\title{
Gelatin-Based Hydrogels Blended with Gellan as an Injectable Wound Dressing
}

\author{
Yueyuan Zheng, ${ }^{\dagger, \S}$ Yuqing Liang, ${ }^{\dagger, \S}$ Depan Zhang, ${ }^{\dagger}$ Xiaoyi Sun, ${ }^{\dagger}$ Li Liang, ${ }^{\ddagger}$ Juan Li, ${ }^{* \dagger \oplus}$
} and You-Nian Liu ${ }^{\dagger}$

${ }^{\dagger}$ College of Chemistry and Chemical Engineering, Central South University, Changsha, Hunan 410083, P. R. China

${ }^{\ddagger}$ State Key Laboratory of Food Science and Technology, School of Food Science and Technology, Jiangnan University, Wuxi, Jiangsu 214122, P. R. China

\section{Supporting Information}

ABSTRACT: Injectable scaffolds are of great interests for skin regeneration because they can fill irregularly shaped defects through minimally invasive surgical treatments. In this study, an injectable hydrogel from biopolymers is developed and its application as wound dressings is examined. Gelatin-based hydrogels were successfully prepared at body temperature upon blending with low content of gellan, and the synergetic effect on the gel formation was carefully characterized through rheological methods. The electrostatic complexation between gelatin and gellan was confirmed to contribute a continuous hydrogel network. The obtained blend hydrogel demonstrates remarkable shear-thinning and self-recovering properties. For

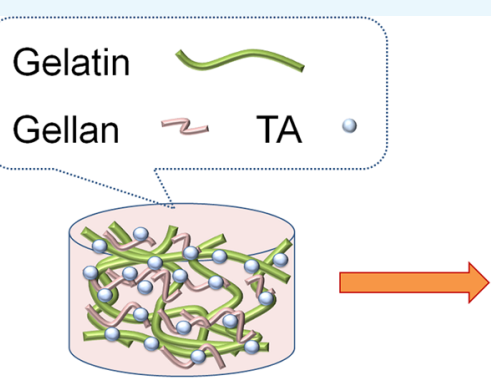

Injectable hydrogel

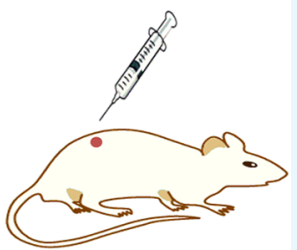

Wound dressing antibacterial purpose, tannic acid was incorporated into the blend hydrogel. In addition, tannic acid-loaded blend hydrogel was verified to accelerate the wound healing on the mice model, significantly than the control groups. Thus, this paper presents a facile approach without chemical modification to construct injectable gelatin-based hydrogels, which have great potential as a wound dressing or tissue scaffold at body temperature.

\section{INTRODUCTION}

Wound healing is a complex and coordinated process, which can be enhanced by a desirable wound dressing material. During the healing processes, the dressing material can absorb excess exudate, provide the moist environment for cell regeneration, protect wounds from bacterial attack, carry healing growth factors, and so on. Various wound dressing materials, such as hydrogels, hydrocolloids, foams, films, and nanoparticles, are commercially available and also being investigated. $^{1-14}$ Among these materials, hydrogels are very attractive for the features of fluid absorbent, physical barrier, and providing a moist scaffold for skin regeneration. ${ }^{13,15}$ However, most hydrogels have poor mechanical properties, slow degradation, and require a secondary dressing to adhere on the skin. ${ }^{13,16}$

Injectable hydrogels with shear-thinning and self-recovering properties are of particular interest due to the unique merits of facile operation, in situ moldability to wound beds, and selfmending their structures and functionalities after damage. ${ }^{1,5,7-10,15,17-23}$ For examples, an injectable gel scaffold with interconnected microporous structure was able to accelerate wound heal by facilitating the cell migration. ${ }^{7}$ The building blocks were composed of peptide RGD-decorated multiarmed poly(ethylene glycol)-vinyl sulfone and two enzyme substrates. ${ }^{7}$ Recently, Guo and co-workers synthesized electroactive injectable hydrogels with hemostasis and adhesiveness based on functionalized chitosan and poly(ethylene glycol) and applied them as wound dressings for cutaneous wound healing. ${ }^{1}$

Gelatin, which is derived from collagen, now is commonly used in tissue engineering due to high abundance, low cost, biocompatibility, biodegradability, and low antigenicity. As wound dressing materials, gelatin contains the peptide sequences for the recognition of integrin receptors in the cells, which are crucial for the cell adhesion. ${ }^{4,24}$ In addition, gelatin tends to form a nanofiber structure, which is essential for skin regeneration. The nanofibers, due to large specific surface area, high porosity, and good permeability, are able to biomimic the extracellular matrix, which favors the cell adhesion, migration, and proliferation. Nevertheless, pristine gelatin has low gelling temperature (below $30^{\circ} \mathrm{C}$ ), which hampers its application at the body temperature. ${ }^{25,26}$ Therefore, gelatin itself is seldom found for skin regeneration. Strategies including chemical modification and physical blending have been adopted to tune the gelling condition of gelatin. For instance, gelatin has been chemically functionalized with unsaturated methacryloyl groups, ${ }^{25}$ thiol groups, ${ }^{4}$ and chemically cross-linked by cross-linking agents, such as genipin,

Received: February 20, 2018

Accepted: April 20, 2018

Published: May 1, 2018 
glutaraldehyde, or periodate-oxidized alginate. ${ }^{3,11,27}$ Compared to chemical modification, physical blending is more appealing due to eco-friendliness, cost-effectiveness, and industrially capable. However, up to now, few reports of gelatin-based injectable blend hydrogels for wound healing have been reported.

In this paper, we introduced an injectable blend hydrogel, based on gelatin and gellan, with excellent shear-thinning as well as self-recovering for wound healing. Gellan, a linear anionic polymer with a tetrasaccharide repeating sequence (residual $\beta$-D-glucose, $\beta$-D-glucuronic acid, and $\alpha$-L-rhamnose in a molar ratio of $2: 1: 1$ ), is approved by the FDA as food additives. It has a gelling behavior, showing a phase diagram dependent of temperature and/or concentration (from solution, fluid "weak gel", to true gel). ${ }^{28-30}$ Investigation on the gelatin behavior of gellan/gelatin binary hydrogel was reported by several works; ${ }^{31-33}$ however, these studies focus on the functional properties for food application. In food industry, gellan is applied as an alternative to gelatin, giving products acceptable by vegetarians and religions that forbid materials from mammals. ${ }^{28}$ Herein, gelatin-based blend hydrogel with very low content of gellan $(\leq 0.5 \%)$ was prepared for wound dressing; the synergetic effect of the gel formation was carefully characterized by rheological methods, and the gelling mechanism was proposed. The shear-thinning and selfrecovering properties of the blend hydrogels were found out. Tannic acid (TA), a kind of polyphenol usually extracted from plants, is biocompatible toward mammalian cells and active against bacteria; however, a high dose of TA on the wounds can cause side effects. ${ }^{34}$ TA was incorporated into the blend hydrogel. Our previous studies found that gelatin-based carrier is suitable for the sustained release of polyphenol compounds. ${ }^{35,36}$ In addition, the effects of blend hydrogel on the wound healing of dorsal skin were evaluated in a mice model. As it turns out, the formulation of TA-loaded blend hydrogel is potent to accelerate wound healing.

\section{EXPERIMENTAL SECTION}

Materials. Gelatin (from porcine skin, Vetec reagent grade, Type A, powder, gel strength $\sim 300$ g Bloom) was purchased from Sigma-Aldrich. Gellan gum (used for plant tissue culture) was obtained from Wako Pure Chemical Industries, Ltd. (Japan). Tannic acid (TA) of analytical reagent was acquired from Aladdin Industrial Corporation. All aqueous solutions were prepared using Millipore water $(18 \mathrm{M} \Omega \mathrm{cm}$, Simplicity Model, Billerica, MA).

Preparation of Blend Hydrogel. Blend hydrogel of gelatin/gellan was prepared by mixing gelatin and gellan powder $(10 \mathrm{wt} \%$ of gelatin concentration and $0.3 \mathrm{wt} \%$ content of gellan) under gentle stirring and heated at $85{ }^{\circ} \mathrm{C}$ in a thermostatic water bath until both biopolymers were completely dissolved. Finally, the solution was cooled at room temperature resulting in the formation of hydrogel.

To incorporate TA into the hydrogels, the hydrogel was first heated, then cooled to about $50{ }^{\circ} \mathrm{C}$, and TA was added under vortex shaking to avoid precipitates. The solution was further cooled down at room temperature after TA was well dispersed. The obtained hydrogel was allowed to stand for $12 \mathrm{~h}$.

Characterization. Morphology of hydrogels was uncovered by scanning electron microscopy (SEM) through a Quanta 250 FEG (FEI, America). Solutions $(20 \mu \mathrm{L})$ obtained by melting the hydrogel were dropped onto a silicon slice and cooled at room temperature to make a gel. Afterward, the sample was lyophilized. Fourier transform infrared (FTIR) spectra were recorded on a Nicolet 510P Fourier transform in the range of $4000-400 \mathrm{~cm}^{-1}$ using $\mathrm{KBr}$ pellets, and the resolution was 2 $\mathrm{cm}^{-1}$. $\zeta$-Potential measurements were performed on a Malvern Zetasizer Nano ZS at $25^{\circ} \mathrm{C}$.

Rheological Properties. Rheological measurements were performed on a rheometer (Anton Paar, MCR 302) with parallel plate geometry ( $25 \mathrm{~mm}$ diameter). The parallel plate was carefully sealed with silicon oil in case of water evaporation during measurement. The gap between the probe and the plate was maintained at $1.0 \mathrm{~mm}$ for the time sweep and frequency sweep experiments. Storage modulus $\left(G^{\prime}\right)$ and loss modulus $\left(G^{\prime \prime}\right)$ as well as the complex viscosity $\left(\left|\eta^{*}\right|\right)$ were measured.

Time sweep experiments were conducted at a constant frequency of $1 \mathrm{~Hz}$ and strain range of $0.01-1000 \%$. The samples were melted at $85{ }^{\circ} \mathrm{C}$ and then about $1.0 \mathrm{~mL}$ of solution was added onto the plate, which was settled at $70^{\circ} \mathrm{C}$, and a program of temperature equilibrium at $37{ }^{\circ} \mathrm{C}$ was first run and then the time-sweep measurement was started.

Frequency sweep experiments were conducted at frequency range of $0.01-10 \mathrm{~Hz}$ and at a strain of $0.1 \%$ and $37{ }^{\circ} \mathrm{C}$.

For the temperature sweep experiment, $G^{\prime}$ and $G^{\prime \prime}$ were monitored as a function of temperature in the range of 10-45 ${ }^{\circ} \mathrm{C}$ at a heating rate of $1.0{ }^{\circ} \mathrm{C} \mathrm{min}{ }^{-1}$. The samples were melted at $85{ }^{\circ} \mathrm{C}$ and then about $1.0 \mathrm{~mL}$ of solution was added onto the plate and settled at $10{ }^{\circ} \mathrm{C}$ for $30 \mathrm{~min}$ to obtain a hydrogel. Afterward, the temperature sweep was conducted at a constant frequency of $1 \mathrm{~Hz}$ and strain of $1.0 \%$ automated controlled by the machine.

Strain sweep was performed on samples from $0.1 \%$ to a maximum strain of $1000 \%$ to determine the limit of the linear viscoelastic region. The frequency was set at $1.0 \mathrm{~Hz}$. The viscosity of the hydrogels was measured as a function of shear rate at $25^{\circ} \mathrm{C}$.

Self-recovering behavior was evaluated by continuous-step strain measurements, alternate-step strains of $0.1 \%$ for $180 \mathrm{~s}$ and large strains $(100,200,300,400$, or $500 \%)$ for 30 s were applied. The frequency was set at $1.0 \mathrm{~Hz}$.

Antibacterial Assay. Antibacterial activity of blend hydrogel was assessed against Escherichia coli, Staphylococcus aureus, and methicillin-resistant $S$. aureus (MRSA) using an agar disc diffusion test. An aliquot of log-phase bacterial stock $\left(10^{5}-10^{6}\right.$ CFU) was uniformly spread on an agar plate. Tested samples were placed on top of the plate, and zones of inhibition were measured after incubation for $12 \mathrm{~h}$ at $37^{\circ} \mathrm{C}$. Sample of TA was prepared by dropping the TA solution $(20 \mu \mathrm{L}, 5 \mathrm{mg} / \mathrm{mL})$ into a sterilized filter paper $(6 \mathrm{~mm}$ in diameter $)$. TA-loaded hydrogels with a diameter of $6 \mathrm{~mm}$ were cut by a tip $(6 \mathrm{~mm}$ in diameter, $1.3 \mathrm{~mm}$ in height, the bulk concentration of TA in the hydrogel is $5 \mathrm{mg} / \mathrm{mL}$ ).

Wound Healing. The in vivo wound-healing effect was estimated on a Balb/c mice model. All animal experiments were performed according to the guidelines, principles, and procedures of the Xiangya Laboratory Animal Center of Central South University (Changsha, China) for the care and use of laboratory animals. The mice were randomly divided into four groups with different treatments $(n=3)$ : phosphatebuffered saline (PBS, $1 \times$, Hyclone), TA $(5 \mathrm{mg} / \mathrm{mL})$, blend hydrogel, and TA-loaded blend hydrogel. The mice, at 6-8 weeks of age, about $20 \mathrm{~g}$, were anesthetized by intraperitoneal injection with $10 \%$ chloral hydrate, and the hair on their backs was shaved. Then, a cross-shape wound was made by a scissor in length of $10 \mathrm{~mm}$ on the dorsal skin back of the tested mice. 
(A)

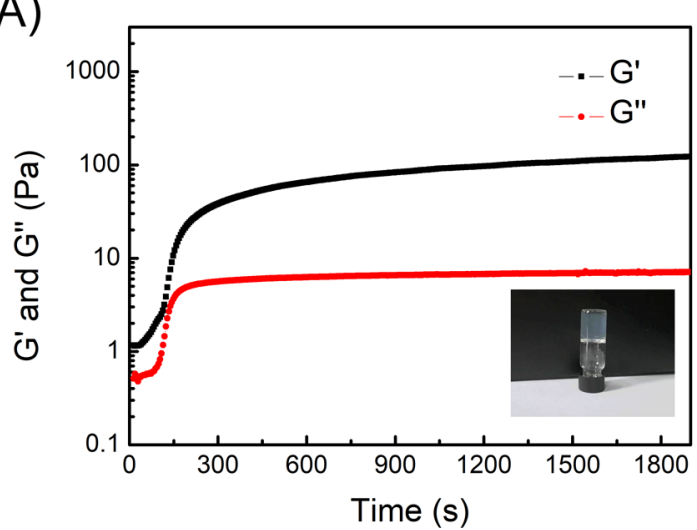

(B)

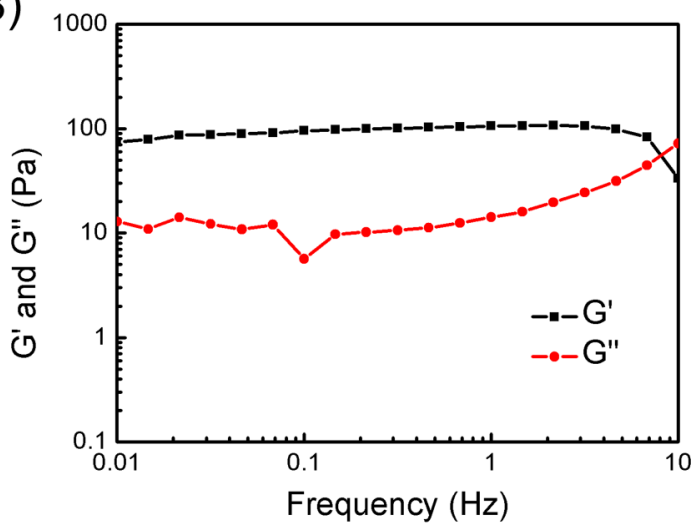

Figure 1. Gelation kinetic (A) and the viscoelastic behavior as a function of frequency (B) of blend hydrogels at $37^{\circ} \mathrm{C}$ by rheological measurement. The inset is the image of blend hydrogel at $37^{\circ} \mathrm{C}$.

Finally, the wounds were added with $20 \mu \mathrm{L}$ of samples using a 26-G syringe without secondary dressing. The wound was photographed by placing a ruler along its side to monitor the process of wound healing. The body weight was also recorded at each 4 days.

\section{RESULTS AND DISCUSSION}

Synergistic Gel Formation of Gelatin/Gellan Mixtures. Synergistic gelation behavior was observed in the mixture of gelatin and gellan at $37{ }^{\circ} \mathrm{C}$. Blends of gelatin and gellan (concentration of gelatin is $10 \mathrm{wt} \%$ and that of gellan $0.3 \mathrm{wt}$ $\%)$ form true gels, which can self-support its own weight at 37 ${ }^{\circ} \mathrm{C}$ (Figure 1, inset image). In contrast, gelatin and gellan alone could not form gels by visualization (Figure S1). It is found that the mixture of gelatin/gellan is able to form gels within the range of $0.05-1.5 \%$ at body temperature. Gellan itself is unable to form gels under the same concentration. However, by increasing the concentration of gellan to $2 \%$, gellan itself can form gels at body temperature. Therefore, the concentration of gellan for blend hydrogel is set at $0.3 \%$, where the noncovalent interaction between gelatin and gellan is preferable.

The gelation kinetics of blend hydrogel at $37^{\circ} \mathrm{C}$ was further investigated following a cooling procedure. As shown in Figure $1 \mathrm{~A}$, at the beginning, the melted solution exhibits a very low moduli $\left(G^{\prime}\right.$ is smaller than $\left.1.0 \mathrm{~Pa}\right)$, though the value of $G^{\prime}$ is larger than $G^{\prime \prime}$. This result is likely to be consistent with the characteristic of weak gel, which is actually a "structure liquid", like normal solutions. ${ }^{28,37}$ However, abrupt increase of $G^{\prime}$ was observed at $150 \mathrm{~s}$, indicating the sol-gel transition. At $300 \mathrm{~s}, G^{\prime}$ increased steadily and reached a plateau at $30 \mathrm{~min}$. The final $G^{\prime}$ of blend hydrogel is 1 magnitude larger than $G^{\prime \prime}$, demonstrating the formation of a true gel. Furthermore, the frequency sweep was performed to evaluate the viscoelastic behavior at constant strain amplitude and within a frequency range of $0.01-10 \mathrm{~Hz}$. The hydrogel is stable and exhibits constant $G^{\prime}$ (linear viscoelastic regime) up to $\sim 5 \mathrm{~Hz}$ (Figure 1B).

Effect of Gellan on the Rheology of Blend Hydrogels. At $10 \%$, gelatin is unable to form gels at body temperature $\left(G^{\prime}\right.$ $\left.<G^{\prime \prime}\right)$, whereas is able to form gels at low temperature $\left(G^{\prime}>\right.$ $G^{\prime \prime}$, Figure 2). The crossover point of $G^{\prime}$ and $G^{\prime \prime}$, termed as the melting temperature, is about $27{ }^{\circ} \mathrm{C}$, as determined by rheometry. For gellan alone, at the low concentration of $0.3 \%$, it shows solution behavior $\left(G^{\prime}<G^{\prime \prime}\right)$ at the temperature ranges studied (Figures $\mathrm{S} 1$ and $\mathrm{S} 2$ ). On addition of gellan into gelatin hydrogels, a small amount of gellan $(<0.5 \%)$ could make

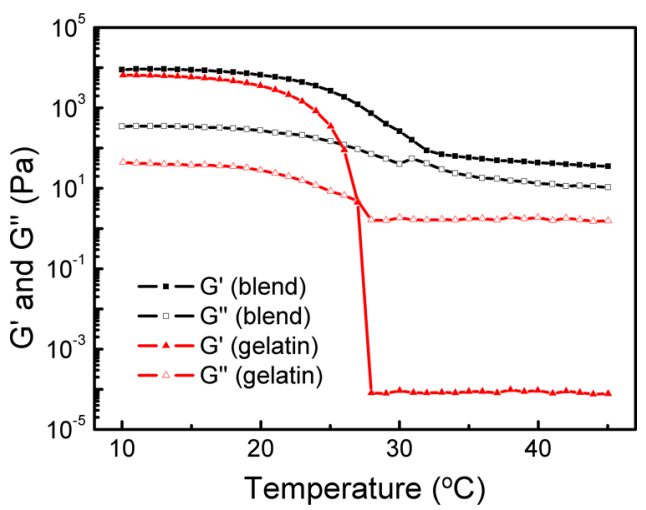

Figure 2. Effect of gellan on $G^{\prime}$ (solid symbols) and $G^{\prime \prime}$ (open symbols) as a function of temperature at a frequency of $1.0 \mathrm{~Hz}$, strain of $0.1 \%$, and a heating rate of $1.0{ }^{\circ} \mathrm{C} / \mathrm{min}$.

a big difference. The obtained blend hydrogels are resistant to the temperature evolution ( $G^{\prime}$ is always larger than $G^{\prime \prime}$ in the range of $10-45^{\circ} \mathrm{C}$ ), indicating that gellan plays an important role in the gelling process of gelatin. It suggests the intermolecular complex formation between gelatin and gellan forming another physical cross-linked network, which is distinct from the network of gelatin alone.

At $37{ }^{\circ} \mathrm{C}, G^{\prime}$ is smaller than $G^{\prime \prime}$ for gelatin hydrogel in the absence of gellan, indicating the solution behavior of gelatin at body temperature. However, $G^{\prime}$ of blend hydrogels is steadily increased with time. At $37^{\circ} \mathrm{C}$, the $G^{\prime}$ of blend hydrogel reaches to $120 \mathrm{~Pa}$ after $30 \mathrm{~min}$ (Figure $1 \mathrm{~A}$ ). The result suggests the network of blend hydrogel is less thermosensitive than the network of gelatin alone.

Shear-Thinning and Self-Recovering Properties of the Hydrogel. Figure $3 \mathrm{~A}$ shows the viscosity of blend hydrogel as a function of shear rate. The viscosity decreases as shear rate elevates. Meanwhile, the shear-thinning behavior is partly reversible after cessation of the shear. Polysaccharide with rigid structure (e.g., xanthan gum and gellan) can be utilized as a modifier for tuning rheological properties of synthetic polymers and biopolymers. ${ }^{2,37,38}$ The rigid polysaccharide endows the hydrogel with desirable shear-thinning properties.

The hydrogels can also be extruded by a $26-\mathrm{G}(0.45 \mathrm{~mm}$ in outer diameter) needle and can be moldable to various patterns (Figure S3). The blend hydrogels reassembled into desired shapes within seconds upon cessation of shear. The result is in 

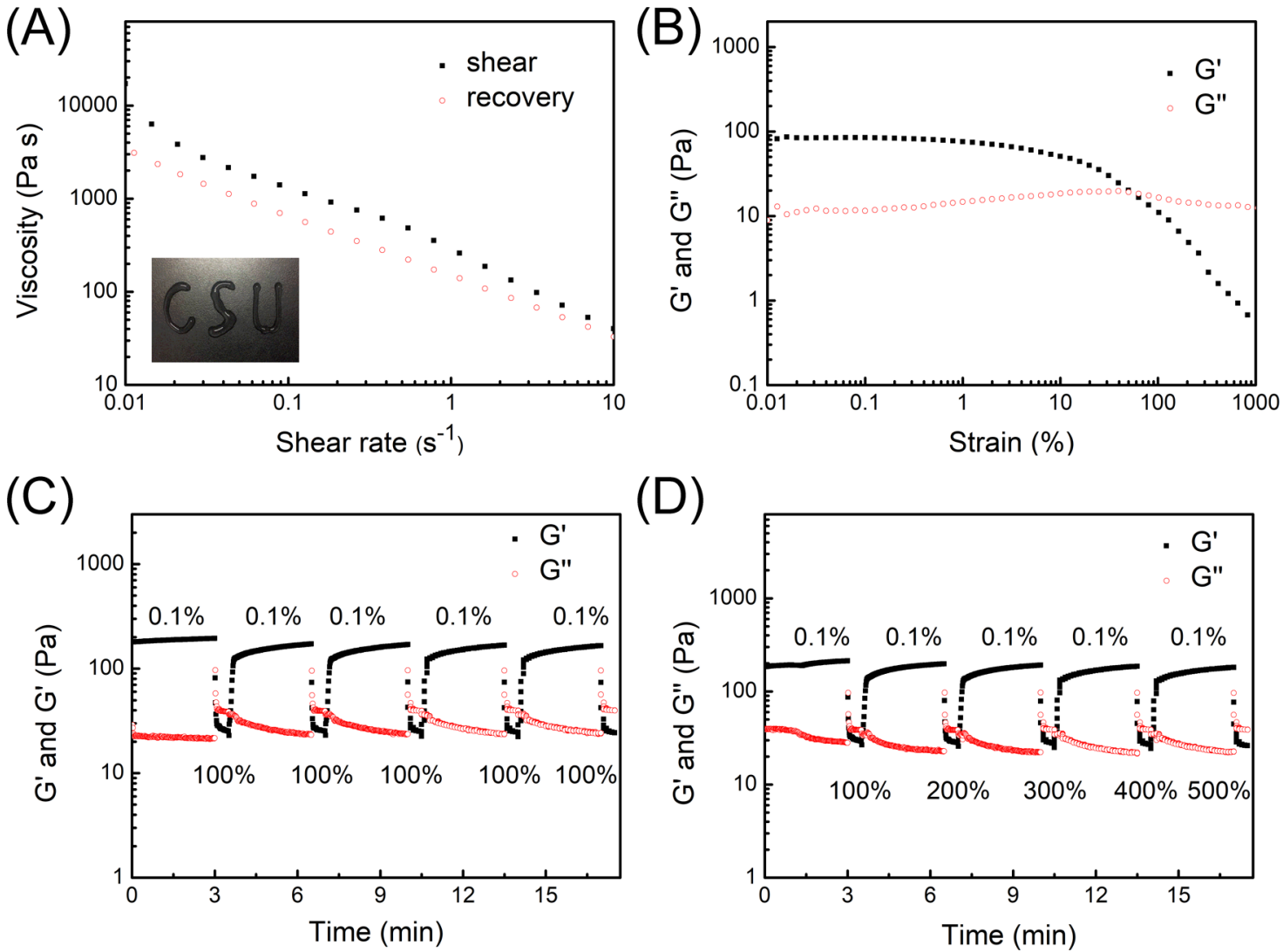

Figure 3. Rheological measurements of blend hydrogel. (A) Viscosity of the blend hydrogel as a function of shear rate at $25^{\circ} \mathrm{C}$. Inset photograph is the patterns of blend hydrogel after injection by a $26-\mathrm{G}$ syringe. The storage and loss moduli of blend hydrogel by continuous- (B) and alternate-step strain sweep $(\mathrm{C}, \mathrm{D})$ at $37^{\circ} \mathrm{C}$.

accordance with the rheological test (Figure 3A). The good shear-thinning and self-healing properties arise from gellan and it can be applied as injectable matrix for cartilage tissue engineering. ${ }^{39-43}$

Furthermore, we assessed the self-recovering of hydrogels by alternate-step strain measurement. As shown in Figure 3B, $G^{\prime}$ of hydrogel is changeless in the strain range of $0.01-10 \%$, whereas $G^{\prime}$ decreases rapidly when the strain is more than $10 \%$ showing that the hydrogel is broken. Hence, high-magnitude strain $(100 \%)$ was applied to damage the hydrogel network for $30 \mathrm{~s}$; afterward, a low-magnitude strain $(0.1 \%)$ was applied to monitor the recovery of the hydrogel structure. A strain deformation of $0.1 \%$ is within the linear viscoelastic range. This process was performed in five cycles, as shown in Figure 3C. Under large strain, $G^{\prime}$ dramatically decreases and becomes smaller than $G^{\prime \prime}$. Notwithstanding, the $G^{\prime}$ restores its strength value immediately after strain changes to $0.1 \%$. In the first cycle, the healing efficiency of $G^{\prime}$ is about $62 \%$ (121 Pa vs initial 194 $\mathrm{Pa}$ ) in first $30 \mathrm{~s}$ and the healing efficiency of $G^{\prime}$ is about $89 \%$ (172 Pa vs initial $194 \mathrm{~Pa}$ ) after a total $180 \mathrm{~s}$. In the following cycles, the healing efficiency of $G^{\prime}$ reaches to $98 \%$ (169 Pa vs initial $172 \mathrm{~Pa}$ ). The data proves that the blend hydrogel has excellent self-healing ability.

Furthermore, by applying increasingly strains (up to 500\%) to damage the hydrogels, the restoring process of $G^{\prime}$ is almost the same as that by constant strains of $100 \%$. Mechanism of self-recovering (also termed as self-healing) relies on the reversibility of the cross-links, which be classified into two approaches, dynamic covalent and noncovalent. In the blend hydrogels, the good self-healing property of blend hydrogels is attributable to the noncovalent bonds of building blocks, such as hydrogen bonds and electrostatic interactions. ${ }^{44}$

Gelation Mechanism. Complexation of gellan with gelatin was investigated by FTIR spectra (Figure 4); most of the bands

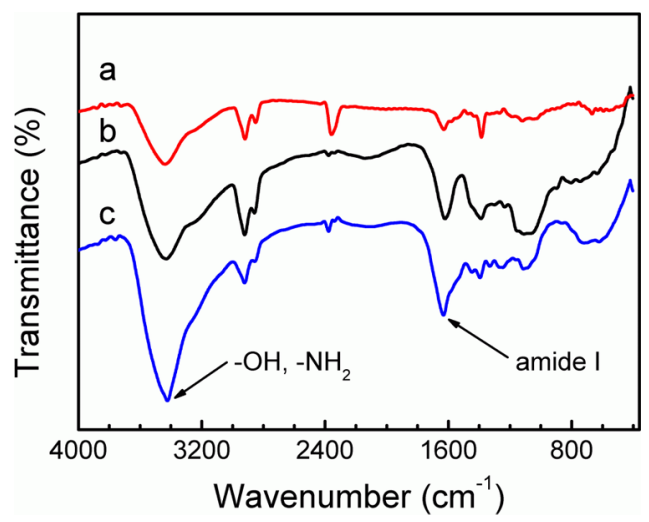

Figure 4. FTIR spectra of gelatin (a), gellan (b), and blend hydrogels (c).

for blend hydrogel are similar as those for gelatin, which supports that gellan is fully blended with gelatin. The peak at $1630 \mathrm{~cm}^{-1}$ for the amide I band of gelatin shifts to $1631 \mathrm{~cm}^{-1}$ after gellan blending, which indicates the occurrence of electrostatic complexation between gellan and gelatin. ${ }^{45}$ In addition, the peak of absorption bands around $3400 \mathrm{~cm}^{-1}$ (contributions of $-\mathrm{OH}$ and $-\mathrm{NH}_{2}$ stretching) was also examined. Blend hydrogel exhibits a lower frequency band 
centered at $3423 \mathrm{~cm}^{-1}$, whereas at $3438 \mathrm{~cm}^{-1}$ and $3434 \mathrm{~cm}^{-1}$ for gelatin and gellan, respectively. The peak shifts to lower wavenumbers due to the dehydration of polymer chains. ${ }^{46,47}$ The results suggest the noncovalent interaction between gelatin and gellan.

To determine the driving force for the formation of blend hydrogels, the $\zeta$-potentials of the two biopolymer solutions were measured as a function of $\mathrm{pH}$ (Figure 5). Gellan displays

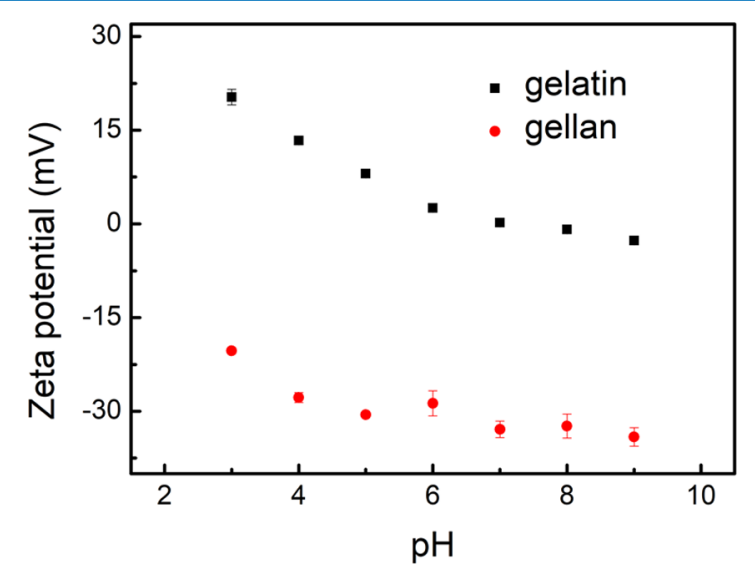

Figure 5. $\zeta$-Potentials of gelatin and gellan as a function of $\mathrm{pH}$.

negative charges over the whole $\mathrm{pH}$ range studied, and the absolute values of $\zeta$-potential gradually decreased as $\mathrm{pH}$ decreased, which can be attributed to protonation of the carboxyl groups of the $\beta$-D-glucuronic acid of gellan molecules. However, the $\zeta$-potential of gelatin is positive when $\mathrm{pH}$ is smaller than 7 and negative when higher than 7 , displaying an isoelectronic point ( $\mathrm{pI}$ ) between $\mathrm{pH} 7$ and $\mathrm{pH}$ 8. The result is in good agreement with the structure information provided by the supplier (gelatin of type A has the pI of 7-9). These results show that gellan and gelatin form electrostatic complexes due to which they have opposite charges at neutral or acidic $\mathrm{pH}$ values.

To understand the electrostatic interaction, blend hydrogel was further prepared at $\mathrm{pH} 4.0$ where the charges of two biopolymers are changed. At $\mathrm{pH} 4.0$, the blend hydrogel become fragile and it is found that the $G^{\prime}$ of the blend hydrogel obtained at $\mathrm{pH} 4.0$ is smaller than that obtained at the initial $\mathrm{pH}$ (Figure S4). At $\mathrm{pH} 4.0$, gelatin carries more positive charges than at the initial $\mathrm{pH}$ (about $\mathrm{pH}$ 5.4), while gellan has less negative charges. By contrast, at acidified $\mathrm{pH}$ closing to the $\mathrm{p} K_{\mathrm{a}}$ ( $\mathrm{p} K_{\mathrm{a}}$ of gellan is about 3.5$){ }^{48}$ and the intra- and intermolecular interaction of gellan itself will increase, which may destroy the complexation of gelatin and gellan. While at $\mathrm{pH}$ values apart from the $\mathrm{p} K_{\mathrm{a}}$, the intermolecular interaction between positively charged gelatin with gellan is dominant, leading to stronger gels.

By using SEM, we observed the blend hydrogel has interconnected porous structures as expected (Figure 6). The low incorporation of gellan doesn't change the morphology of gelatin hydrogels. The blend hydrogels are made of nanofibers with pore size of $2-3 \mu \mathrm{m}$. Nanofiber-based wound dressings have drawn great attention due to their merits of large specific surface area, high porosity, and good permeability, which promoting cell adhesion migration and proliferation. ${ }^{6,49}$ The porous structure is beneficial for rapid diffusion of nutrients throughout the network, improving the efficacy in cell attachment, proliferation, and migration. ${ }^{7,50,51}$

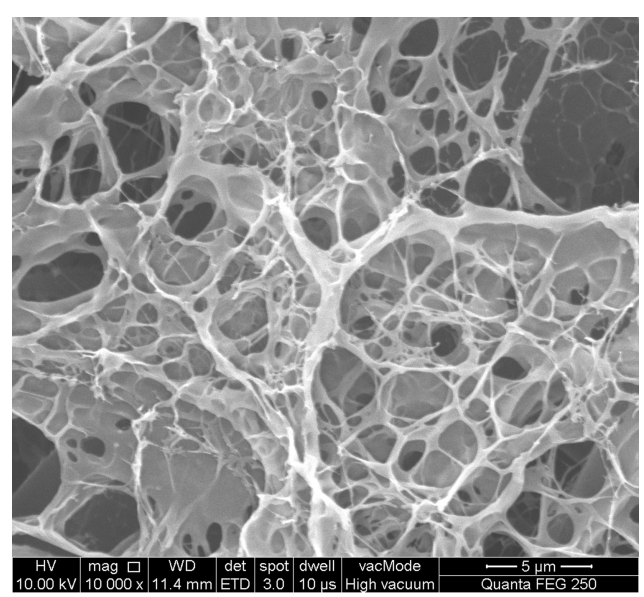

Figure 6. SEM image of the blend hydrogel.

Influence of TA on the Rheology Properties of Hydrogel. Recent studies showed protein-polysaccharide electrostatic hydrogels are of interest to assemble functional gels due to the method not requiring any denaturation or chemical cross-linking as opposed to protein hydrogels. ${ }^{52}$ In our studies, each component alone is unable to form gels, whereas the blends of them do form gels under the experimental conditions. Thus, the blend hydrogels have great potential to develop as functional biomaterials to incorporate bioactive molecules.

TA was directly incorporated into the blend hydrogel during the cooling process close to $50{ }^{\circ} \mathrm{C}$. TA has strong binding with gelatin. Homogeneous mixing is finely controlled by vortex shaking to avoid precipitates. $G^{\prime}$ of blend hydrogel after TA loading is higher than before due to the cross-linking effect of TA (Figure S5). Furthermore, the shear-thinning properties are also well maintained (Figure S5). To understand the supramolecular interaction of TA with gellan and gelatin, we attempted to cross-link the two polymers by TA. Nevertheless, it is found that neither gelatin (10\%) nor gellan $(0.3 \%)$ is able to form hydrogels at $37{ }^{\circ} \mathrm{C}$ in the presence of $\mathrm{TA}$ up to a concentration of $40 \mathrm{mg} / \mathrm{mL}$ (Figure S6). The results suggest that the binding between TA and gelatin or gellan cannot destroy the main structure of the blend hydrogel, probably due to the low mass ratio of TA to gelatin $(1: 20)$. In our studies, a small ratio of TA is applied to avoid macroscopic separation, similar to films composed of gelatin and TA. ${ }^{53}$ Previously, we found that soluble polydisperse particles are formed at low ratios of tea polyphenol to gelatin. ${ }^{36}$ It further suggests that the blend hydrogel is an excellent reservoir for other active substances, and cells as well.

Antibacterial Properties. An ideal wound dressing should protect the wound bed from pathogenic bacteria. The biopolymer-based hydrogel is fragile to bacteria, which can induce inflammation and cause unfavorable smell due to fermentation. TA exhibits a broad antibacterial spectrum against either Gram-negative bacteria or Gram-positive bacteria. The bacterial growth can be inhibited by TA using a multiple mode of action involving destabilization of the cytoplasmic membrane, inhibition of extracellular microbial enzymes, deprivation of the substrates required for microbial growth or direct action on microbial metabolism through inhibition of oxidative phosphorylation, and so on ${ }^{54-56}$ Hence, herbal medicine TA can reduce the occurrence of antibacterial resistance, which is a superiority over chemical antibiotics. 
(A)

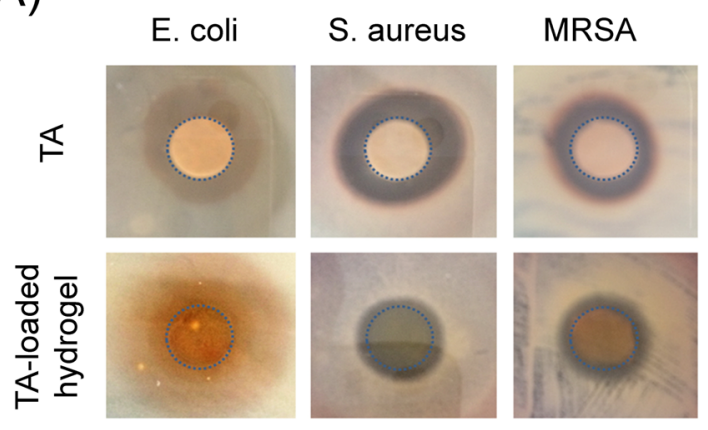

(B)

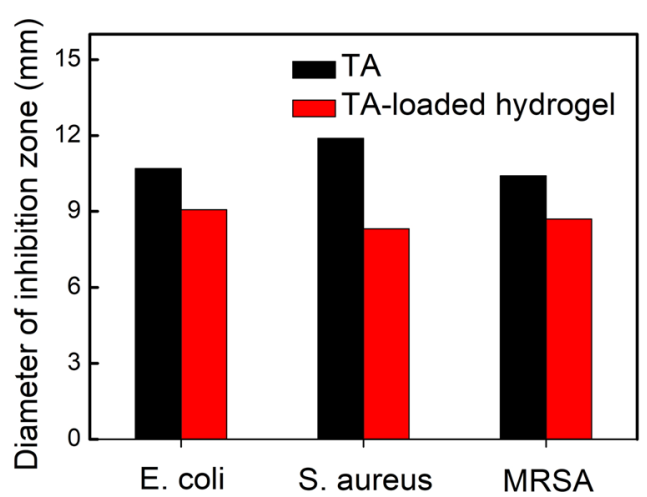

Figure 7. (A) Photographs of agar diffusion test result for TA and TA-loaded blend hydrogel $(5 \mathrm{mg} / \mathrm{mL})$ against E. coli, S. aureus, and MRSA; the dotted circles depict the original size of TA-loaded filter paper or blend hydrogel. (B) The diameter of inhibition zone for TA and TA-loaded hydrogel against E. coli, S. aureus, and MRSA.

(A)

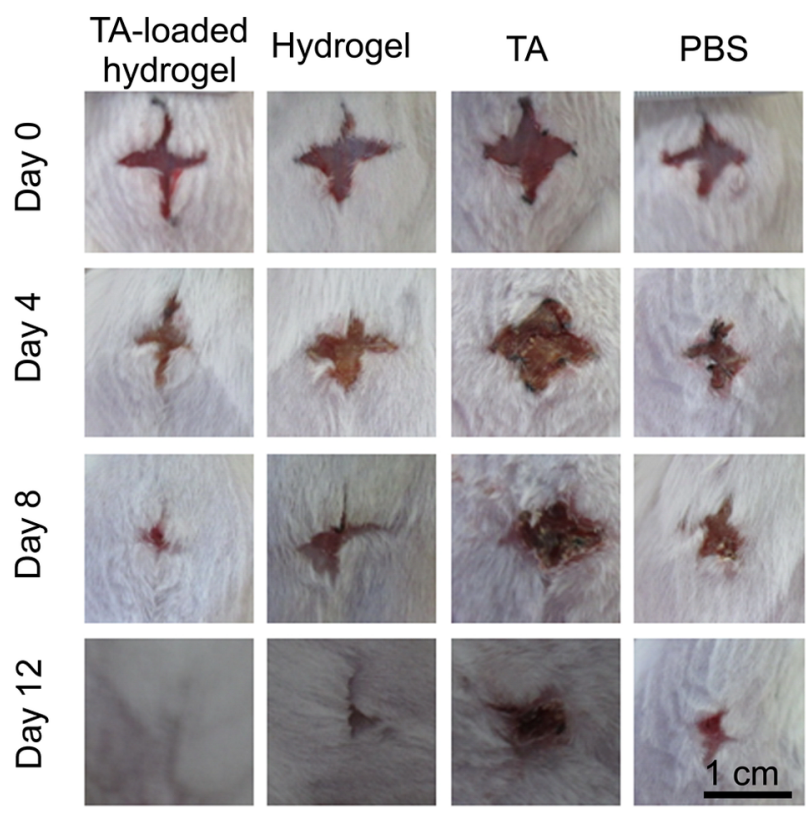

(B)
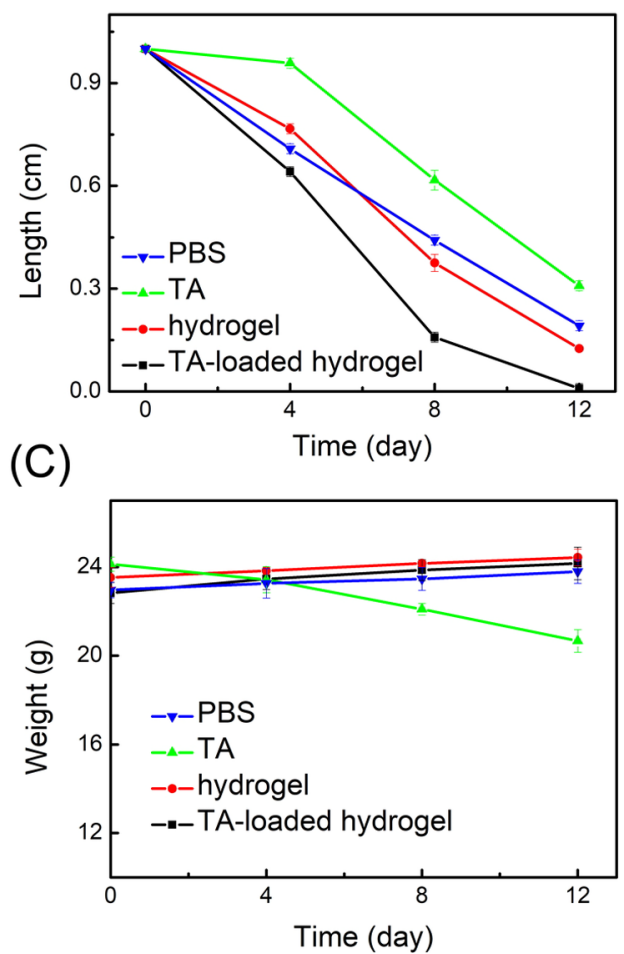

Figure 8. TA-loaded blend hydrogel promotes wound healing in a Balb/c mouse model. (A) Representative images of wound closure during 12 day experiments. Quantification of wound closure (B) and body weight (C) of mice during the treatment.

Herein, TA-loaded blend hydrogel was prepared and its antibacterial activities were evaluated by the agar disc diffusion assay. Gram-negative bacterium (E. coli), Gram-positive bacterium ( $S$. aureus), and drug-resistant bacteria (MRSA) were used as model bacteria. The results showed that the TAloaded hydrogel inhibits the growth of all bacteria including $S$. aureus, E. coli, and MRSA, although the inhibition zone of TAloaded hydrogel is smaller than that of TA solution (Figure 7). As a negative control, blend hydrogel has no inhibition activity on bacteria growth. The results indicate that TA was sustained released from the blend hydrogel and exerted long-term antibacterial efficacy. To verify the sustained release property of TA-loaded hydrogel, in vitro release was performed using a transdermal diffusion apparatus according to the paper ${ }^{57}$ and described in Supporting Information. The result confirms that
TA-loaded hydrogel has a sustained release of TA (Figure S7). It is reported that complexation of polyphenols with gelatin can afford adequate protection for gelatin against microbial degradation, which plays an important role in the tanning industry. ${ }^{58}$ Owing to the antibacterial properties, the blend hydrogel can reduce the growth of bacteria and prolong the shelf-life in storage and half-life for therapy.

Wound Healing. The wound healing efficacy of the hydrogels was evaluated in a mice model. Four groups of mice (treatment by PBS, TA, hydrogel, and TA-loaded hydrogel) were tested. The wounds were created into fullthickness cross-shape with about $10 \mathrm{~mm}$ in length. The wounds were treated by the test samples only once and without any other dressing put on the skin across the whole experiments. On observation days, the wounds were recorded (Figure 8A). 


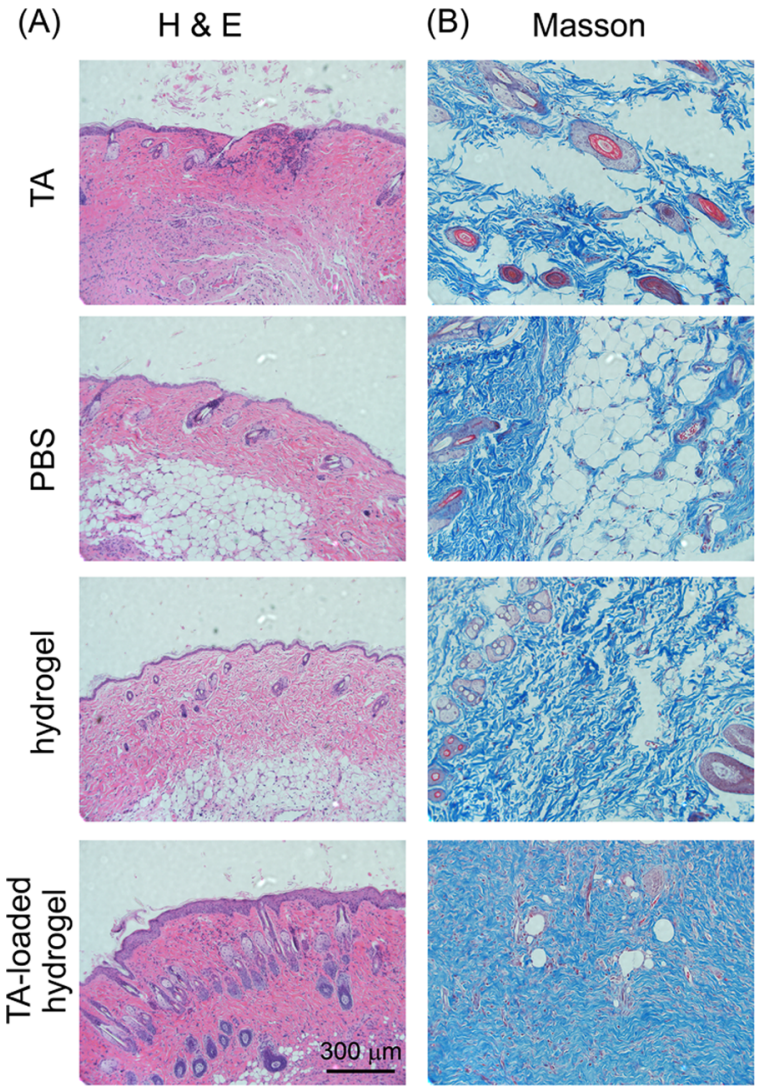

(C)

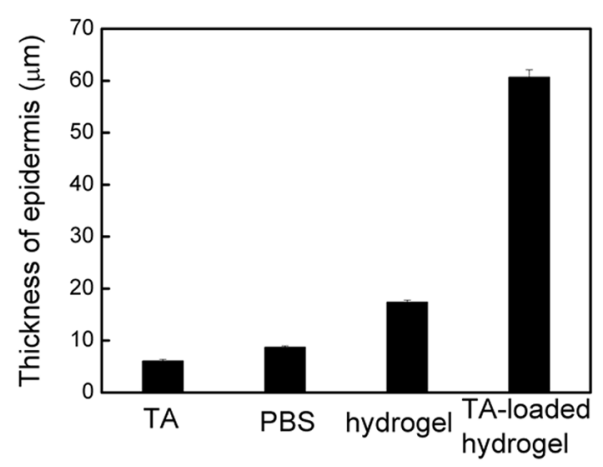

(D)

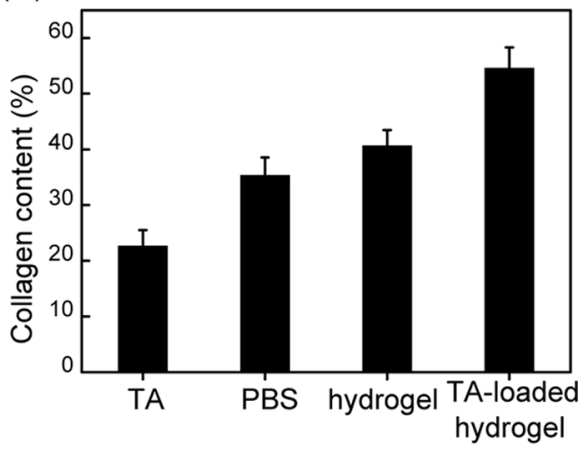

Figure 9. H\&E (A) and Masson's trichrome (B) staining, thickness of epidermis (C), and collagen content (D) of TA-loaded hydrogel group at 12 day wound healing.

The change of length of wounds and body weight was plotted as a function of time, as shown in Figure 8B,C. For TA-loaded hydrogel group, the wounds reduced remarkably and completely diminished on the 12th day. No scar was found, and the skin was fully covered with hair. On the 12th day, the healing rate is $100 \%$ in terms of the decrease ratio of the length of wounds. The area of wound closure was also measured; TAloaded hydrogel group has a fast recovery in the index of area compared with other groups (Figure S8).

In contrast, the group treated by TA recovers slowly. On the 12th day, the healing rate was $71 \%$ and scar was still observed. Furthermore, an inflammatory response was indicated by observing fluids exuded from the wounds. Also, the body weight of mice treated by TA was gradually decreased. Total tannins from Entada phaseoloides (L.) Merr. were reported exerting a wound-healing promoting effect, and the concentration of tannins is about $9.38-37.50 \mu \mathrm{g} / \mathrm{mL}$. When the concentration was higher than $37.5 \mu \mathrm{g} / \mathrm{mL}$, tannins inhibited the collagen synthesis in NIH3T3 cells. ${ }^{34}$ In this paper, $5.0 \mathrm{mg} /$ $\mathrm{mL}$ of TA is applied as control. High concentration of TA on the one hand could promote wound shrinkage but on the other hand could hinder the healing rate by inhibiting the collagen synthesis; the results were consistent with those observed. ${ }^{34}$ In Figure S8, the area of wound of TA group on the 4th day was even larger than the beginning, which probably results from the negative effect of high concentration of TA.

For PBS group, on the 12th day, the healing rate is about $81 \%$ in terms of the length ratio of wounds. Saline can promote the healing process in part by the faster contraction, similar to the phenomena found in the literature. ${ }^{12}$ For the TA-free blend hydrogel group, the healing process was faster in comparison to the PBS group. In our study, blend hydrogel is more efficacious than PBS for healing (healing rate is $88 \%$ on the 12 th day for blend hydrogel), implying the role of hydrogel matrix, where gelatin has a well-established effect on cell migration and proliferation. ${ }^{4,24}$ Therefore, TA-loaded hydrogel not only provides a sustained drug delivery for minimizing the adverse effect of TA, but also facilitates skin repair in a cooperative way.

In hematoxylin and eosin ( $\mathrm{H} \& \mathrm{E})$ and Masson's trichrome staining, the epidermal thickness, skin tissue formation, and collagen deposition were evaluated (Figure 9). In TA-loaded hydrogel group, epidermal thickness was $60 \mu \mathrm{m}$, which was significantly higher than other groups. Scoring results of wound granulation tissue formation were $5,4,5$, and 8 for groups of PBS, TA, hydrogel, and TA-loaded hydrogel, respectively. TAloaded hydrogel group scores the highest value in the granulation tissue formation. In the TA-loaded hydrogel group, collagen fibers with parallel arrangement are more accumulated and collagen content is also higher than other groups. In addition, more hair follicles are observed. The results indicated that TA-loaded hydrogel group has very effective skin regeneration and function restoration over other groups.

To gain insight of wound healing, we examined the cell cytotoxicity and cell migration of blend hydrogels. L929 cells treated by blend hydrogel are growing better than the control groups, showing higher cell viability (Figure S9), suggesting the good biocompatibility of blend hydrogels. Furthermore, cell proliferation and migration are revealed across the scratched area by microscopy images after $24 \mathrm{~h}$ incubation (Figure 10). The cells in the control group are more confluent than those in the blend hydrogel group. The migration of the cells occurs when the hydrogel is gradually dissolved during incubation. 


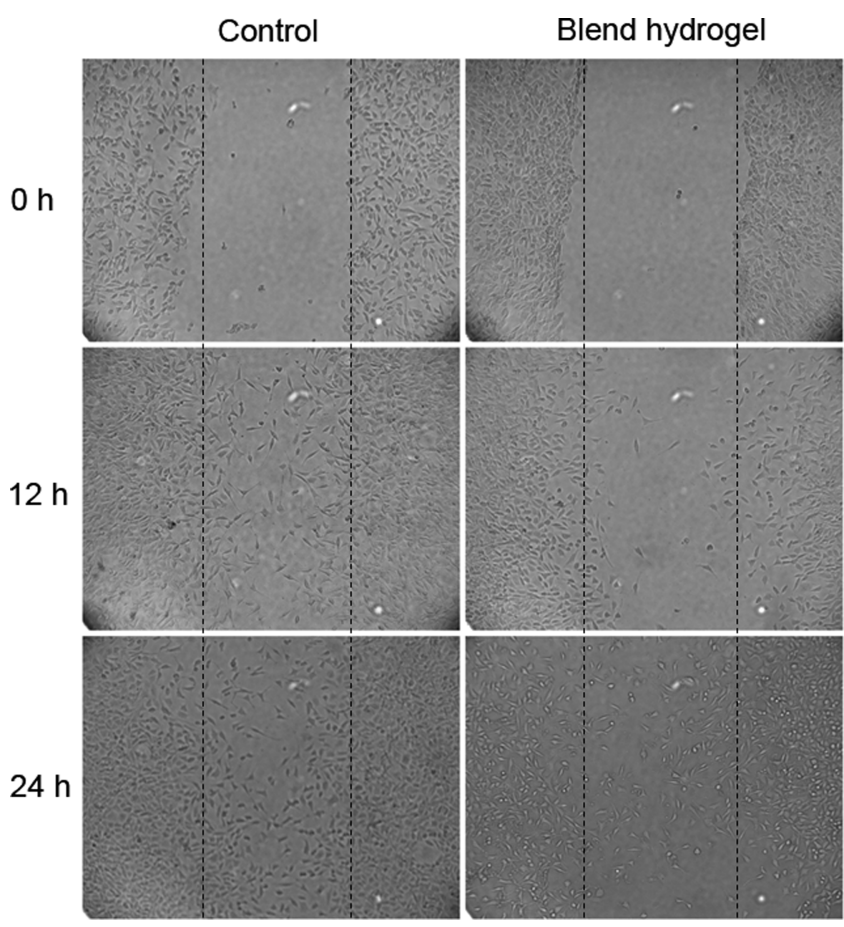

Figure 10. Cell migration assay of blend hydrogel for 0,12 , and $24 \mathrm{~h}$. The dotted lines indicate the wound scratch created using a pipette tip.

The in vitro results imply that the blend hydrogel can be slowly degraded in vivo, and the erosion can create space for cell growth and migration.

The investigation of wound healing by blend hydrogel is preliminary, and we further found the hydrogel did not show the same effect on a round-shape of full-thick wounds (data not shown). The hydrogel gradually lost water and adhered fiercely on the wound beds during the healing, which delayed the healing process to some extent. The obstacles might be circumvented by spaying saline or water, or covering with other occlusive dressings.

\section{CONCLUSIONS}

Blending with gellan is suitable to make gelatin a true gel under body temperature. The mechanism of gelation is mainly attributed to the electrostatic complexation. In particular, the blend hydrogel exhibits good injectability with self-recovering property. The blend formulation not only improves the thermal gelation property of gelatin, but also makes dressings capable of fully conforming to wound beds. The blend hydrogel is beneficial for sustained delivery of TA and promoting cell adhesion and migration, supported by animal experiments. Altogether, this unique blend hydrogel has a bright future for wound healing and/or tissue engineering.

\section{ASSOCIATED CONTENT}

\section{S Supporting Information}

The Supporting Information is available free of charge on the ACS Publications website at DOI: 10.1021/acsomega.8b00308.

Experimental for in vitro release, cell cytotoxicity, migration, H\&E, and Masson's trichrome staining; photographs of gelatin, gellan, and their mixtures at 37 ${ }^{\circ} \mathrm{C}$ (Figure S1); $G^{\prime}$ and $G^{\prime \prime}$ of gellan alone as a function of temperature (Figure S2); different moldable shapes of blend hydrogels after injection (Figure S3); $G^{\prime}$ and $G^{\prime \prime}$ of blend hydrogel prepared at $\mathrm{pH} 4.0$ (Figure S4); shearthinning property of TA-loaded blend hydrogel (Figure S5); physical mixture of gellan/TA and gelatin/TA (Figure S6); sustained release of TA-loaded hydrogel (Figure S7); data for area of wound closure (Figure S8); cell cytotoxicity (Figure S9) (PDF)

\section{AUTHOR INFORMATION}

\section{Corresponding Author}

*E-mail: juanli@csu.edu.cn. Tel/Fax:+86-731-88879616.

ORCID

Xiaoyi Sun: 0000-0002-1407-6108

Juan Li: 0000-0002-1979-9049

You-Nian Liu: 0000-0002-7078-5937

\section{Author Contributions}

${ }^{\S}$ Y.Z. and Y.L. contributed equally to this work.

\section{Notes}

The authors declare no competing financial interest.

\section{ACKNOWLEDGMENTS}

This work was financially supported by the National Natural Science Foundation of China (Nos. 21574147 and 21374133), the Innovation-Driven Project of Central South University (No. 2017CX020), and the Postgraduate Science Foundation of Central South University (No. 2016zzts240).

\section{REFERENCES}

(1) Zhao, X.; Wu, H.; Guo, B.; Dong, R.; Qiu, Y.; Ma, P. X. Antibacterial Anti-oxidant Electroactive Injectable Hydrogel as Selfhealing Wound Dressing with Hemostasis and Adhesiveness for Cutaneous Wound Healing. Biomaterials 2017, 122, 34-47.

(2) Tran, N. Q.; Joung, Y. K.; Lih, E.; Park, K. D. In Situ Forming and Rutin-Releasing Chitosan Hydrogels As Injectable Dressings for Dermal Wound Healing. Biomacromolecules 2011, 12, 2872-2880.

(3) Balakrishnan, B.; Mohanty, M.; Umashankar, P. R.; Jayakrishnan, A. Evaluation of an In Situ Forming Hydrogel Wound Dressing based on Oxidized Alginate and Gelatin. Biomaterials 2005, 26, 6335-6342.

(4) Dong, Y.; Sigen, A.; Rodrigues, M.; Li, X.; Kwon, S. H.; Kosaric, N.; Khong, S.; Gao, Y.; Wang, W.; Gurtner, G. C. Injectable and Tunable Gelatin Hydrogels Enhance Stem Cell Retention and Improve Cutaneous Wound Healing. Adv. Funct. Mater. 2017, 27, No. 1606619

(5) Zhao, L.; Niu, L.; Liang, H.; Tan, H.; Liu, C.; Zhu, F. pH and Glucose Dual-Responsive Injectable Hydrogels with Insulin and Fibroblasts as Bioactive Dressings for Diabetic Wound Healing. ACS Appl. Mater. Interfaces 2017, 9, 37563-37574.

(6) Li, Z.-W.; Li, C. W.; Wang, Q.; Shi, S. J.; Hu, M.; Zhang, Q.; Cui, H. H.; Sun, J. B.; Zhou, M.; Wu, G. L.; Dang, J. Z.; Lu, L. C. The Cellular and Molecular Mechanisms Underlying Silver Nanoparticle/ Chitosan Oligosaccharide/Poly(vinyl alcohol) Nanofiber-Mediated Wound Healing. J. Biomed. Nanotechnol. 2017, 13, 17-34.

(7) Griffin, D. R.; Weaver, W. M.; Scumpia, P. O.; Di Carlo, D.; Segura, T. Accelerated Wound Healing by Injectable Microporous Gel Scaffolds Assembled from Annealed Building Blocks. Nat. Mater. 2015, $14,737-744$.

(8) Nimal, T. R.; Baranwal, G.; Bavya, M. C.; Biswas, R.; Jayakumar, R. Anti-staphylococcal Activity of Injectable Nano Tigecycline/ Chitosan-PRP Composite Hydrogel Using Drosophila melanogaster Model for Infectious Wounds. ACS Appl. Mater. Interfaces 2016, 8, 22074-22083.

(9) Zhang, Z.; He, T.; Yuan, M.; Shen, R.; Deng, L.; Yi, L.; Sun, Z.; Zhang, Y. The In Situ Synthesis of Ag/Amino Acid Biopolymer Hydrogels as Mouldable Wound Dressings. Chem. Commun. 2015, 51, 15862-15865. 
(10) Xu, W.; Song, Q. A.; Xu, J. F.; Serpe, M. J.; Zhang, X. Supramolecular Hydrogels Fabricated from Supramonomers: A Novel Wound Dressing Material. ACS Appl. Mater. Interfaces 2017, 9, 11368-11372.

(11) Wu, S.; Deng, L.; Hsia, H. S.; Xu, K.; He, Y.; Huang, Q. R.; Peng, Y.; Zhou, Z. H.; Peng, C. Evaluation of Gelatin-Hyaluronic Acid Composite Hydrogels for Accelerating Wound Healing. J. Biomater. Appl. 2017, 31, 1380-1390.

(12) Svensjö, T.; Pomahac, B.; Yao, F.; Slama, J.; Eriksson, E. Accelerated Healing of Full-Thickness Skin Wounds in a Wet Environment. Plast. Reconstr. Surg. 2000, 106, 602-612.

(13) Rivera, A. E.; Spencer, J. M. Clinical Aspects of Full-Thickness Wound Healing. Clin. Dermatol. 2007, 25, 39-48.

(14) Rangasamy, S.; Tak, Y. K.; Kim, S.; Paul, A.; Song, J. M. Bifunctional Therapeutic High-Valence Silver-Pyridoxine Nanoparticles with Proliferative and Antibacterial Wound-Healing Activities. J. Biomed. Nanotechnol. 2016, 12, 182-196.

(15) Zhang, Y. S.; Khademhosseini, A. Advances in Engineering Hydrogels. Science 2017, 356, No. eaaf3627.

(16) Menaker, G. M. Wound Dressings at the Turn of the Millennium. Curr. Probl. Dermatol. 2001, 13, 86-89.

(17) Yu, L.; Ding, J. Injectable Hydrogels as Unique Biomedical Materials. Chem. Soc. Rev. 2008, 37, 1473-1481.

(18) Taylor, D. L.; Panhuis, M. I. H. Self-Healing Hydrogels. Adv. Mater. 2016, 28, 9060-9093.

(19) Zhang, Y.; Yang, B.; Xu, L.; Zhang, X.; Tao, L.; Wei, Y. Selfhealing Hydrogels Based on Dynamic Chemistry and Their Biomedical Applications. Acta Chim. Sin. 2013, 71, 485-492.

(20) Tseng, T. C.; Tao, L.; Hsieh, F. Y.; Wei, Y.; Chiu, I. M.; Hsu, S. H. An Injectable, Self-Healing Hydrogel to Repair the Central Nervous System. Adv. Mater. 2015, 27, 3518-3524.

(21) Loebel, C.; Rodell, C. B.; Chen, M. H.; Burdick, J. A. Shearthinning and Self-healing Hydrogels as Injectable Therapeutics and for 3D-Printing. Nat. Protoc. 2017, 12, 1521-1541.

(22) Liao, J. F.; Wang, B. Y.; Huang, Y. X.; Qu, Y.; Peng, J. R.; Qian, Z. Y. Injectable Alginate Hydrogel Cross-Linked by Calcium Gluconate Loaded Porous Microspheres for Cartilage Tissue Engineering. ACS Omega 2017, 2, 443-454.

(23) Liao, J. F.; Jia, Y. P.; Wang, B. Y.; Shi, K.; Qian, Z. Y. Injectable Hybrid Poly( $\varepsilon$-caprolactone)-b-poly(ethylene glycol)-b-poly( $\varepsilon$-caprolactone) Porous Microspheres/Alginate Hydrogel Cross-linked by Calcium Gluconate Crystals Deposited in the Pores of Microspheres Improved Skin Wound Healing. ACS Biomater. Sci. Eng. 2018, 4, 1029-1036.

(24) Lee, S. B.; Jeon, H. W.; Lee, Y. W.; Lee, Y. M.; Song, K. W.; Park, M. H.; Nam, Y. S.; Ahn, H. C. Bio-artificial Skin Composed of Gelatin and $(1 \rightarrow 3),(1 \rightarrow 6)-\beta$-Glucan. Biomaterials 2003, 24, 25032511.

(25) Loessner, D.; Meinert, C.; Kaemmerer, E.; Martine, L. C.; Yue, K.; Levett, P. A.; Klein, T. J.; Melchels, F. P. W.; Khademhosseini, A.; Hutmacher, D. W. Functionalization, Preparation and Use of Cellladen Gelatin Methacryloyl-based Hydrogels as Modular Tissue Culture Platforms. Nat. Protoc. 2016, 11, 727-746.

(26) Klouda, L. Thermoresponsive Hydrogels in Biomedical Applications A Seven-Year Update. Eur. J. Pharm. Biopharm. 2015, 97, 338-349.

(27) Bertoni, F.; Barbani, N.; Giusti, P.; Ciardelli, G. Transglutaminase reactivity with gelatine: Perspective applications in tissue engineering. Biotechnol. Lett. 2006, 28, 697-702.

(28) Morris, E. R.; Nishinari, K.; Rinaudo, M. Gelation of Gellan - A Review. Food Hydrocolloids 2012, 28, 373-411.

(29) Kang, D.; Zhang, H.; Katsuyoshi, N. Gellan Gum and <tepcommon:author-query $>A Q 1$ : Please provide a DOI number for ref 29 or indicate if one doesn\&amp;\#x2019;t exist.</tep-common:authorquery $>$ Modified Gellan Gum Hydrogels as Biomedical Materials. Prog. Chem. 2014, 26, 1172-1189.

(30) Osmałek, T.; Froelich, A.; Tasarek, S. Application of Gellan Gum in Pharmacy and Medicine. Int. J. Pharm. 2014, 466, 328-340.
(31) Lau, M. H.; Tang, J.; Paulson, A. T. Texture Profile and Turbidity of Gellan/Gelatin Mixed Gels. Food Res. Int. 2000, 33, 665671.

(32) Lee, K. Y.; Shim, J.; Lee, H. G. Mechanical Properties of Gellan and Gelatin Composite Films. Carbohydr. Polym. 2004, 56, 251-254.

(33) Pranoto, Y.; Lee, C. M.; Park, H. J. Characterizations of Fish Gelatin Films Added with Gellan and kappa-Carrageenan. LWT-Food Sci. Technol. 2007, 40, 766-774.

(34) Su, X.; Liu, X.; Wang, S.; Li, B.; Pan, T.; Liu, D.; Wang, F.; Diao, Y.; Li, K. Wound-healing Promoting Effect of Total Tannins from Entada phaseoloides (L.) Merr. in Rats. Burns 2017, 43, 830-838.

(35) Zhang, L.; Qi, Z.; Huang, Q.; Zeng, K.; Sun, X.; Li, J.; Liu, Y.-N. Imprinted-like Biopolymeric Micelles as Efficient Nanovehicles for Curcumin Delivery. Colloids Surf., B 2014, 123, 15-22.

(36) Zhou, H.; Sun, X.; Zhang, L.; Zhang, P.; Li, J.; Liu, Y.-N. Fabrication of Biopolymeric Complex Coacervation Core Micelles for Efficient Tea Polyphenol Delivery via a Green Process. Langmuir 2012, 28, 14553-14561.

(37) Liu, Z.; Yao, P. Injectable Thermo-responsive Hydrogel Composed of Xanthan Gum and Methylcellulose Double Networks with Shear-thinning Property. Carbohydr. Polym. 2015, 132, 490-498.

(38) Le, X. T.; Turgeon, S. L. Rheological and Structural Study of Electrostatic Cross-linked Xanthan Gum Hydrogels Induced by betaLactoglobulin. Soft Matter 2013, 9, 3063-3073.

(39) Oliveira, J. T.; Picciochi, R.; Santos, T. C.; Martins, L.; Pinto, L. G.; Malafaya, P. B.; Sousa, R. A.; Marques, A. P.; Castro, A. G.; Mano, J. F.; Neves, N. M.; Reis, R. L. Injectable Gellan Gum Hydrogels as Supports for Cartilage Tissue Engineering Applications. Tissue Eng., Part A 2008, 14, 748.

(40) Douglas, T. E. L.; Schietse, J.; Zima, A.; Gorodzha, S.; Parakhonskiy, B. V.; Khalenkow, D.; Shkarin, R.; Ivanova, A.; Baumbach, T.; Weinhardt, V.; Stevens, C. V.; Vanhoorne, V.; Vervaet, C.; Balcaen, L.; Vanhaecke, F.; Slosarczyk, A.; Surmeneva, M. A.; Surmenev, R. A.; Skirtach, A. G. Novel Self-gelling Injectable Hydrogel/alpha-Tricalcium Phosphate Composites for Bone Regeneration: Physiochemical and Microcomputer Tomographical Characterization. J. Biomed. Mater. Res., Part A 2018, 106, 822-828.

(41) Du, H.; Hamilton, P.; Reilly, M.; Ravi, N. Injectable In Situ Physically and Chemically Crosslinkable Gellan Hydrogel. Macromol. Biosci. 2012, 12, 952-961.

(42) Posadowska, U.; Brzychczy-Wloch, M.; Drozdz, A.; KrokBorkowicz, M.; Wlodarczyk-Biegun, M.; Dobrzynski, P.; Chrzanowski, W.; Pamula, E. Injectable Hybrid Delivery System Composed of Gellan Gum, Nanoparticles and Gentamicin for the Localized Treatment of Bone Infections. Expert Opin. Drug Delivery 2016, 13, $613-620$.

(43) Oliveira, J. T.; Santos, T. C.; Martins, L.; Picciochi, R.; Marques, A. P.; Castro, A. G.; Neves, N. M.; Mano, J. F.; Reis, R. L. Gellan Gum Injectable Hydrogels for Cartilage Tissue Engineering Applications: In Vitro Studies and Preliminary In Vivo Evaluation. Tissue Eng., Part A 2010, 16, 343-353.

(44) Li, Q. W.; Liu, C. L.; Wen, J. R.; Wu, Y. Z.; Shan, Y.; Liao, J. F. The Design, Mechanism and Biomedical Application of Self-healing Hydrogels. Chin. Chem. Lett. 2017, 28, 1857-1874.

(45) Wu, T.; Huang, J.; Jiang, Y.; Hu, Y.; Ye, X.; Liu, D.; Chen, J. Formation of Hydrogels Based on Chitosan/Alginate for the Delivery of Lysozyme and their Antibacterial Activity. Food Chem. 2018, 240, 361-369.

(46) Lai, H.; Wu, P. A Infrared Spectroscopic Study on the Mechanism of Temperature-induced Phase Transition of Concentrated Aqueous Solutions of Poly(N-isopropylacrylamide) and NIsopropylpropionamide. Polymer 2010, 51, 1404-1412.

(47) Bridelli, M. G.; Stani, C.; Bedotti, R. Fourier Transform Infrared Conformational Investigation of Type I Collagen Aged by In Vitro Induced Dehydration and Non-enzymatic Glycation Treatments. J. Biol. Res. 2017, 90, 45-50.

(48) Picone, C. S. F.; Da Cunha, R. L. Interactions between Milk Proteins and Gellan Gum in Acidified Gels. Food Hydrocolloids 2010, 24, 502-511. 
(49) Lee, S. J.; Heo, D. N.; Lee, D.; Heo, M.; Rim, H.; Zhang, L. G.; Park, S. A.; Do, S. H.; Moon, J. H.; Kwon, I. K. One-Step Fabrication of AgNPs Embedded Hybrid Dual Nanofibrous Oral Wound Dressings. J. Biomed. Nanotechnol. 2016, 12, 2041-2050.

(50) De France, K. J.; Xu, F.; Hoare, T. Structured Macroporous Hydrogels: Progress, Challenges, and Opportunities. Adv. Healthcare Mater. 2018, 7, No. 1700927.

(51) Wang, Q.; Xu, J. X.; Jin, H. M.; Zheng, W. H.; Zhang, X. L.; Huang, Y. X.; Qian, Z. Y. Artificial Periosteum in Bone Defect RepairA Review. Chin. Chem. Lett. 2017, 28, 1801-1807.

(52) Le, X. T.; Rioux, L. E.; Turgeon, S. L. Formation and Functional Properties of Protein-Polysaccharide Electrostatic Hydrogels in Comparison to Protein or Polysaccharide Hydrogels. Adv. Colloid Interface Sci. 2017, 239, 127-135.

(53) Peña, C.; De La Caba, K.; Eceiza, A.; Ruseckaite, R.; Mondragon, I. Enhancing Water Repellence and Mechanical Properties of Gelatin Films by Tannin Addition. Bioresour. Technol. 2010, 101, 6836-6842.

(54) Serrano, J.; Puupponen-Pimia, R.; Dauer, A.; Aura, A.-M.; SauraCalixto, F. Tannins: Current Knowledge of Food Sources, Intake, Bioavailability and Biological Effects. Mol. Nutr. Food Res. 2009, 53, S310-S329.

(55) Scalbert, A. Antimicrobial Properties of Tannins. Phytochemistry 1991, 30, 3875-3883.

(56) Widsten, P.; Cruz, C. D.; Fletcher, G. C.; Pajak, M. A.; Mcghie, T. K. Tannins and Extracts of Fruit Byproducts: Antibacterial Activity against Foodborne Bacteria and Antioxidant Capacity. J. Agric. Food Chem. 2014, 62, 11146-11156.

(57) Zhou, W.; He, S. Y.; Yang, Y. J.; Jian, D.; Chen, X.; Ding, J. S. Formulation, Characterization and Clinical Evaluation of Propranolol Hydrochloride Gel for Transdermal Treatment of Superficial Infantile Hemangioma. Drug Dev. Ind. Pharm. 2015, 41, 1109-1119.

(58) Davies, R. I.; Coulson, C. B.; Lewis, D. A. Polyphenols in Plant, Humus, and Soil. iii. Stabilization of Gelatin by Polyphenol Tanning. J. Soil Sci. 1964, 15, 299-309. 